TecnoLógicas

ISSN-p 0123-7799

ISSN-e 2256-5337

Vol. 23, No. 47, pp. 77-91

Enero-abril de 2020

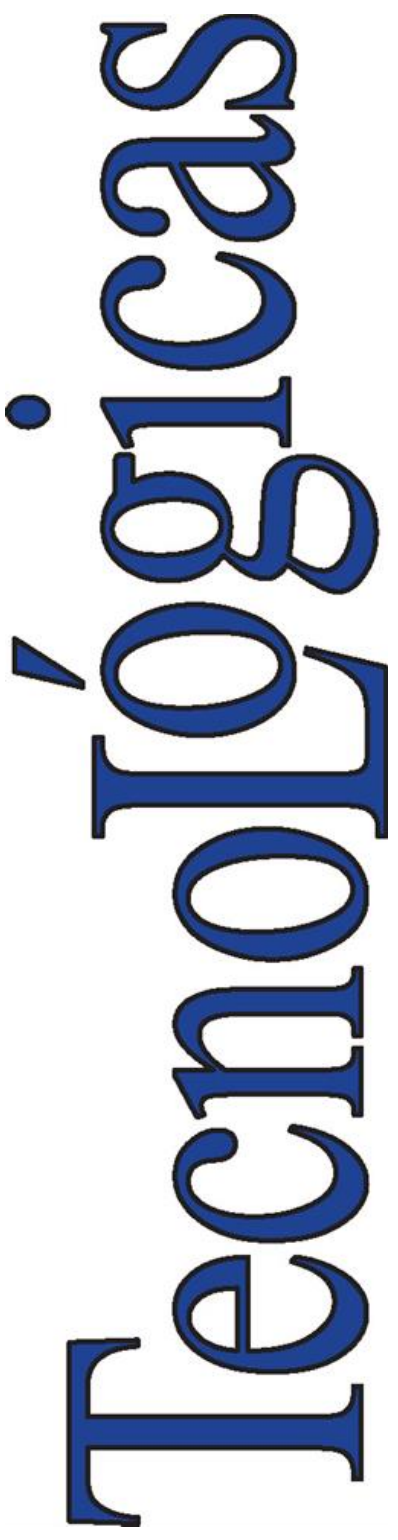

(C) Instituto Tecnológico Metropolitano Este trabajo está licenciado bajo una Licencia Internacional Creative Commons Atribución (CC BY-NC-SA)

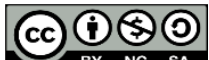

Artículo de Investigación/Research Article

\section{Estimación del riesgo ambiental causado por plaguicidas en cultivos de arveja de Ipiales, Nariño-Colombia}

\section{Estimation of the Environmental Risk Caused by Pesticides in Pea Crops of Ipiales, Nariño-Colombia}

\author{
David Álvarez-SáncheziD 1 , \\ Diana Chaves-Morillo(D2, \\ Eyder Gómez-López ${ }^{\text {D }}{ }^{3}$ y \\ Andrés Hurtado-Benavides (D) 4
}

Recibido: 27 de junio de 2019 Aceptado: 04 de octubre de 2019
MSc. Ciencias Agrarias, Facultad de Ciencias Agrícolas, Universidad de Nariño, Pasto-Colombia, daealvarezsa@udenar.edu

2 Ingeniera Agroindustrial, Facultad de Ingeniería Agroindustrial, Universidad de Nariño, Pasto-Colombia,dmchavesm@udenar.edu.co

3 PhD. Biotecnología, Facultad de Ciencias Agropecuarias, Universidad Nacional de Colombia-Sede Palmira, Cali-Colombia, eydgomezlo@unal.edu.co

4 PhD. Ciencia y Tecnología de los Alimentos e Ingeniería, Facultad de Ingeniería Agroindustrial, Universidad de Nariño, Pasto-Colombia, ahurtadob@udenar.edu.co 


\section{Resumen}

En el municipio de Ipiales, departamento de Nariño, se concentra la mayor área cultivada con arveja (Pisum sativum) de Colombia, con cerca de 3.070 ha, en donde se ha identificado un alto uso de plaguicidas asociado al establecimiento del modelo productivo. Por tanto, el objetivo de este estudio es evaluar el riesgo de impacto ambiental que genera la aplicación de los principales fungicidas, insecticidas, herbicidas $\mathrm{y}$ bactericidas. Para ello, se consideraron tres grupos de campesinos, previamente identificados como C1, C2 y C3 con una muestra de diez fincas por grupo. En una parcela de cada finca se determinó la materia orgánica, $\mathrm{pH}$, textura, densidad aparente, distancia a fuentes hídricas y se caracterizaron los plaguicidas usados en dos ciclos de cultivo de arveja. La información recolectada fue ingresada al sistema de soporte IIRAmb para estimar el indicador I-PHY, el cual determinó el impacto ambiental de los plaguicidas. Los resultados sugieren la presencia de 44 ingredientes activos, con un uso promedio finca de $25,1 \mathrm{~kg} / \mathrm{ha} / \mathrm{año}$; el indicador I-PHY global presentó diferencias estadísticas significativas entre C1 $(4,04)$ respecto a C2 $(5,06)$ y C3 $(5,28)$, estos últimos grupos, mostraron el mayor riesgo ambiental principalmente por el tipo de moléculas usadas y las condiciones en las cuales se desarrolló el cultivo. A partir de estos resultados se genera una herramienta de monitoreo y evaluación, con el fin de mejorar las intervenciones en el sistema para incrementar el nivel de sustentabilidad.

\section{Palabras clave}

Pisum sativum, contaminación, plaguicidas, indicadores ambientales.

\section{Abstract}

The Municipality of Ipiales (Department of Nariño, Colombia) concentrates the largest area cultivated with pea (Pisum sativum L.) in the country, nearly 3.070 ha, where an extensive use of pesticides has been associated with the implementation of a productionbased model. Therefore, the objective of this study is to evaluate the environmental impact of the application of the most common fungicides, insecticides, herbicides, and bactericides. For that purpose, 30 farms were divided into three groups of ten farms each: $\mathrm{C} 1, \mathrm{C} 2$, and $\mathrm{C} 3$. The organic matter, $\mathrm{pH}$, texture, apparent density, and distance to water sources of the plot of each farm were determined, and the pesticides used in the two cultivation cycles of pea were characterized. The collected information was entered into a support system, IIRAmb, in order to estimate the indicator, I-PHY, which determined the environmental impact of the pesticides. The results suggest the presence of 44 active ingredients, with an average use per farm of $25.1 \mathrm{~kg} / \mathrm{ha} /$ year. The global I-PHY indicator presented significant statistical differences between C1 (4.04), C2 (5.06), and C3 (5.28). The last two groups showed the highest environmental risk, especially because the type of molecules they used and the conditions under which the crops developed. These results can be used to create a monitoring and evaluation tool in order to improve the adjustments to the system and increase its sustainability.

\section{Keywords}

Pisum sativum, contamination, pesticides, environmental indicators. 


\section{INTRODUCCIÓN}

La estrategia de «modernización» agrícola, que prevalece desde la década de 1960 bajo el paradigma de la «Revolución verde», se sustenta en la lógica del control de las condiciones ambientales, a través de la simplificación de los elementos de un sistema, remplazándolos por insumos sintéticos. Otorgando, en la mayoría de los casos, el máximo nivel de rendimiento sin considerar las externalidades inherentes, generadas por el uso y dependencia de «paquetes tecnológicos».

En este sentido, uno de los elementos de este modelo que recurrentemente es señalado por las implicaciones negativas que genera, es el uso de plaguicidas, que hacen parte de las recomendaciones que siguen los campesinos como estrategia productiva para combatir los problemas sanitarios de los cultivos. Su empleo, ha incrementado el riesgo potencial de contaminación afectando la calidad del suelo, el agua y el aire; en consecuencia, la generación de impactos crónicos sobre la salud humana, la flora y la fauna [1]-[3].

Agrava esa situación el hecho que en la última década ha sido evidente la pérdida de efectividad de estos productos, induciendo al uso de mayores dosis e intervalos más cortos para obtener el mismo resultado, ampliando el desbalance causado. Autores como Altieri y Nicholls [4], señalan que la agricultura actual se basa en insumos, más no en procesos, lo cual, en síntesis, limita los mecanismos de regulación ecológica.

Le Bellec et al. [2] y Grenón et al. [5], ante la complejidad de esta problemática y la falta de información, sugieren recurrir a indicadores que permitan estimar el riesgo y sintetizar datos y variables de ingreso, en función de la sustentabilidad del sistema; entendiendo que el estudio de la dinámica de uso de los productos fitosanitarios puede considerarse como una estrategia para buscar alternativas a los problemas generados.
$\mathrm{Al}$ respecto, el índice agroecológico IPHY (presión fitosanitaria) se destaca por ser un indicador predictivo, basado en la lógica difusa que evalúa los riesgos del uso de plaguicidas en la agricultura e identifica los principales impactos ambientales sobre cuatro componentes: agua subterránea, agua de superficie, aire y riesgo global [6].

Las variables con las cuales opera, están relacionadas con las características del ingrediente activo, el contexto agroambiental de la parcela (condiciones climáticas, características del suelo y desarrollo de la parcela) y las condiciones de aplicación [2], [7].

El carácter sistémico de I-PHY fue decisivo para abordar el estudio del sistema productivo de arveja desarrollado en el sur de Nariño (Colombia), especialmente en el municipio de Ipiales, en el cual trabajos preliminares sugieren una alta dependencia de plaguicidas relacionada con la adopción de variedades con mayor potencial de rendimiento, introducidas desde finales de la década de 1990 en la región [8]. Con lo cual, esta investigación inició con la recolección de datos a través de una serie de entrevistas y estimaciones sobre el uso de estos productos fitosanitarios en fincas dedicadas al cultivo, con el fin de comprobar la hipótesis de riesgo ambiental vinculado al sistema.

Para ello se usó una tipificación multicriterio desarrollada en la región, en la cual se definieron tres variaciones de agricultura convencional, que pueden incidir, por sus particularidades, sobre el uso de plaguicidas y el impacto generado.

$\mathrm{El}$ primer clúster $\mathrm{C} 1$, asociado a campesinos con mayor capital, alta inversión de insumos y mecanización del suelo, a diferencia de los clústeres C2 y C3, que incluyen, entre otros criterios, a pequeños propietarios de tierra, menor inversión en insumos, empleo de mano de obra familiar, y para $\mathrm{C} 3$, adicionalmente, se considera la topografía de ladera [8]. 
Por lo anterior, en este trabajo se evaluó el riesgo de impacto ambiental de plaguicidas usados en cultivos de arveja en el municipio de Ipiales a través del indicador I-PHY, con el objetivo de generar una herramienta de monitoreo y evaluación que permita mejorar las intervenciones en el sistema para incrementar el nivel de sustentabilidad.

\section{MATERIALES Y MÉTODOS}

La investigación se desarrolló durante dos ciclos de cultivo, estudiados entre febrero y diciembre de 2017, en 30 fincas dedicadas al cultivo de arveja del municipio de Ipiales, departamento de Nariño (Colombia). Este municipio se seleccionó por ser el principal productor a nivel nacional [9].

La zona de estudio fue identificada como bosque seco montano bajo (Bs-MB) con temperatura promedio de $11,9^{\circ} \mathrm{C}$, precipitación media anual de $1.110 \mathrm{~mm}$ y humedad relativa de 81 a $84 \%$ [10]. Del universo de fincas productoras, fue seleccionada una muestra representativa de diez fincas por clúster. Teniendo en cuenta la tipificación previa realizada en la zona, las principales características de los grupos se resumen así [8]:

$-\mathrm{C} 1$ = tamaño de finca entre 1 y 2 ha; área dedicada al cultivo de arveja de 1 a 5 ha debido a la práctica de arrendamiento de lotes; topografía ondulada a quebrada; costo de producción $>\$ 9$ millones/ha; rendimiento 6,6 a 8,2 t/ha.

-C2 = tamaño de finca entre 0.5 y $1 \mathrm{ha}$; área dedicada al cultivo de arveja de 0,3 a 0,6 ha; topografía plana a ondulada; costos de producción de $\$ 5$ y $\$ 7$ millones $/$ ha; rendimiento de 4,9 a 6,6 t/ha.

-C3 = tamaño de finca entre 0,5 y 1 ha; área dedicada al cultivo de arveja de 0,5 a 1 ha; topografía quebrada; costos de producción de $\$ 7$ y $\$ 9$ millones/ha; rendimiento de 4,9 t/ha.

\subsection{Componentes agroambientales}

Con el fin de establecer los insumos para el cálculo del indicador I-PHY, se tomaron diez submuestras de suelo conformando una muestra homogénea a la cual se le determinó las variables: materia orgánica mediante el método WalkleyBlack, $\mathrm{pH}$ mediante el método potenciométrico, textura mediante el método de Bouyuocos y densidad aparente mediante probeta graduada.

Paralelamente, en el lote se calculó la pendiente y la distancia desde la parcela de arveja a la fuente hídrica más cercana $(\mathrm{m})$.

\subsection{Caracterización de plaguicidas}

Mediante una entrevista semiestructurada y verificación de envases y empaques, se registraron los principales productos plaguicidas usados en el cultivo de arveja, además, durante cada semestre se acompañó al productor por lo menos una vez en una jornada de aplicación.

-La base de datos consignó la siguiente información: tipo de plaguicida (fungicida, insecticida, herbicida, bactericida, otro); ingrediente activo; frecuencia de aplicación expresada en días; dosis del producto en kilogramos o litros sobre área y categoría toxicológica (I, II, III, IV de acuerdo con la Organización Mundial de la Salud -OMS-).

El uso total de insumos se calculó mediante la ecuación propuesta por Bravo et al. [11], extrapolando los resultados a términos de ingrediente activo por hectárea y año (1):

$$
\mathrm{a} / \mathrm{h} a / \mathrm{año}=\frac{\frac{P_{f}}{A_{p}} * \% \text { i.a. } * \frac{N A_{p}}{C} * \frac{N C}{A} * H}{H_{t}}
$$

Dónde, $\mathrm{Pf}=$ producto formulado $\mathrm{kg} \mathrm{o} \mathrm{Ll}$; $\mathrm{Ap}=$ frecuencia de aplicación; \% i.a. $=\%$ ingrediente activo; $\mathrm{NAp}=$ número de aplicaciones; $\mathrm{C}=$ ciclo; $\mathrm{NC}=$ número de 
ciclos; $\mathrm{A}=$ año; $\mathrm{H}=$ área aplicada; $\mathrm{Ht}=$ área total cultivada.

\subsection{Cálculo del indicador I-PHY}

Por medio de este indicador se estimó el riesgo de impacto ambiental por el uso de plaguicidas en la parcela, con una escala cualitativa de 0 a 10 . El puntaje se construyó mediante agregaciones sucesivas de 28 variables en cuatro módulos de riesgo, de acuerdo con las reglas de decisión definidas por Van der Werf y Zimmer [7]: agua subterránea (Iphysubterr), agua de superficie (Iphy-superf), aire (Iphy-aire) y riesgo global (Iphyglobal). En la Fig. 1 adaptada de Le Bellec [2], se representa la estructura del indicador.

Los valores de los módulos se calcularon mediante el sistema de soporte de decisión IIRAmb (Índices Integrados de Riesgo Ambiental) desarrollado para
Microsoft Excel® [5], ingresando el resultado de la caracterización de los plaguicidas que relacionó las propiedades fisicoquímicas y ecotoxicológicas de los productos aplicados (Koc, DT $50, \mathrm{DL}_{50}, \mathrm{Ksp}$ ), los componentes agroambientales (pendiente, suelo y agua) y las características de aplicación (dosis, frecuencia y tipo de aplicación). Se consideró que valores entre 0 a 3 presentan un riesgo bajo o nulo de impacto ambiental, 3 a 5 riesgo moderado, 5 a 8 riesgo alto y mayores a 8 de riesgo muy alto.

\subsection{Análisis estadístico}

Cada módulo de I-PHY fue comparado a partir del resultado de las diez fincas de cada clúster, desarrollando un análisis de varianza y pruebas de comparación de medias LSD-Fischer $(\alpha=0,05)$ usando Statgraphics Centurión XVI.

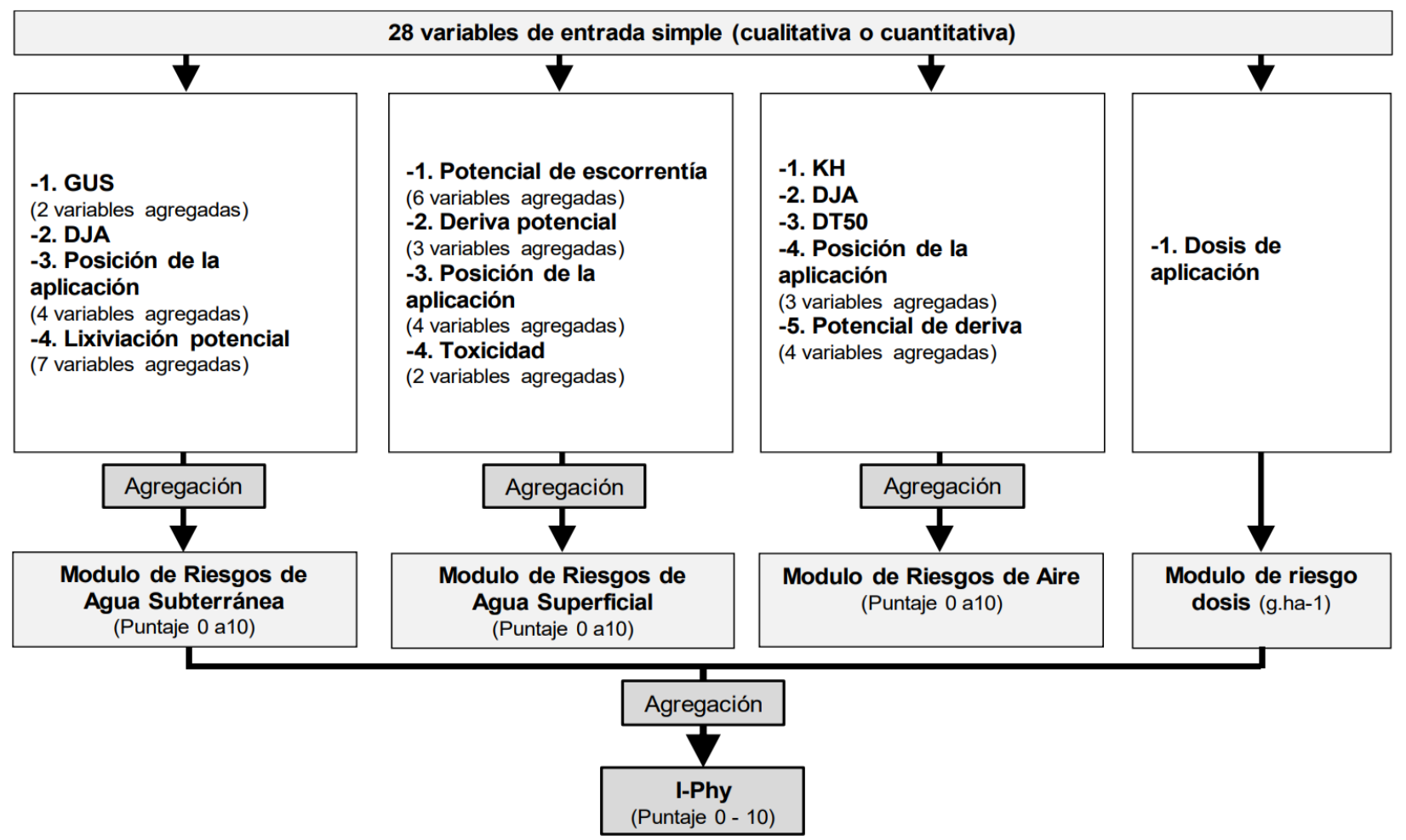

Fig. 1. Estructura del indicador I-PHY por agregación de variables. Fuente: [2]. 


\section{RESULTADOS Y DISCUSIÓN}

\subsection{Componentes agroambientales}

Como indica Arregui et al. [12], el indicador I-PHY no solo se basa en las características fisicoquímicas de los plaguicidas usados, sino que posibilita la integración de variables de riesgo relacionadas con las condiciones de la parcela. Por tanto, el sistema de cultivo, el tipo de suelo y el relieve afectan la predicción del riesgo potencial [7].

En esta investigación, debido a la característica de la tipificación previa, que integraba información topográfica y técnico-productiva, los parámetros estimados presentaron valores similares dentro de los clústeres, tal como se muestra en la Tabla 1.

De forma general, los resultados anteriores coincidieron con los valores reportados en estudios realizados en el municipio de Ipiales por Mena et al. [13].

El contenido de materia orgánica se catalogó como medio ( $5-10 \%)$ con acidez moderada para $\mathrm{C} 1$ y $\mathrm{C} 2$, a diferencia de una mayor proporción de suelos fuertemente ácidos para $\mathrm{C} 3$, relacionados a contenidos superiores de material orgánico (Tabla 1).

Predominó la clase textural francoarcillo-arenoso en el $73 \%$ de las fincas, con especial significado por la relación del movimiento del agua y retención de humedad. Asociado a este parámetro, la densidad aparente presentó un rango entre 0,70 y $0,94 \mathrm{~g} \mathrm{~cm}^{-3}$ concordando con Jaramillo [14], quien indica que los suelos andisoles se comportan de forma característica con densidades por debajo a $0,9 \mathrm{~g} \mathrm{~cm}^{-3}$, y el uso de tractor para la preparación de la parcela podría explicar la variación encontrada entre clústeres.

De igual forma, la tipificación jugó un papel importante en la estimación de la pendiente, que como indica Beber et al. [6], junto a la distancia a la fuente hídrica, son factores determinantes que incrementan el indicador I-PHY y cada uno de sus módulos. Las fincas agrupadas en C2 presentaron un valor promedio entre 3 a $10 \%$ (pendiente ligera a moderada) y los clústeres $\mathrm{C} 1$ y $\mathrm{C} 3$ presentaron un promedio superior, con valores entre 8 a $25 \%$ (pendiente moderada a fuerte).

La distancia del cultivo a una fuente hídrica no mostró una tendencia definida, ya que depende de la disposición de la parcela y la geografía local. Sin embargo, se destaca que en diferentes casos el valor estimado fue menor a quince metros, con lo cual se presentó un riesgo inminente de contaminación [6].

\subsection{Caracterización de plaguicidas}

En la muestra evaluada se registró un total de 67 productos plaguicidas comerciales, de estos, se identificaron 44 ingredientes activos descritos en la Tabla 2 y Tabla 3. En las fincas, se utilizó por parte del campesino hasta 28 productos para un ciclo de cultivo, donde el $59 \%$ correspondió a fungicidas, el $29 \%$ a insecticidas, el $8 \%$ a herbicidas y el $5 \%$ a bactericidas.

La frecuencia de aplicación tuvo un régimen calendarizado dependiente del clima, siendo 5 días el periodo de aplicación en temporada de lluvia y de 8 a 15 días en temporada seca.

En cuanto a la toxicidad de los plaguicidas se estableció que el $50 \%$ corresponde a la categoría II (moderadamente peligrosos), el $43 \%$ a la categoría III (poco peligrosos) y el $7 \%$ a la categoría I (muy peligrosos); por otro lado, del total de ingredientes activos usados, el Instituto Colombiano Agropecuario, entidad encargada de la regulación de este tipo de productos, estableció la cancelación o revaluación del registro de venta a 11 , ya que su toxicidad no supera los estándares mínimos en prevención y mitigación de daños a la salud humana y al medio ambiente [15].

Durante el período de estudio, el uso de ingredientes activos de alto riesgo fue habitual, desconociendo por parte de los campesinos las limitaciones e 
implicaciones de su aplicación. Los vacíos en las medidas regulatorias, la falta de información y la presión de la industria, hacen que este tipo de productos se adquieran sin que sus efectos nocivos sean obstáculos que limiten su comercialización.
Por el volumen empleado sobresalen los ingredientes activos fungicidas clorotalonil, difenoconazol y metalaxil, todos de categoría toxicológica II, considerados como potenciales agentes contaminantes de agua subterránea y degradación lenta (Tabla 2).

Tabla 1. Resultados de algunos parámetros fisicoquímicos de suelo por clúster. Fuente: elaboración propia.

\begin{tabular}{lcccccc}
\hline \multicolumn{1}{c}{ Parámetro } & Clúster 1 (C1) & $\mathbf{S D}^{*}$ & Clúster 2 (C2) & $\boldsymbol{S D}$ & Clúster 3 (C3) & $\boldsymbol{S D}$ \\
\hline Materia & 5,78 & $\pm 1,26$ & 5,69 & $\pm 1,13$ & 6,14 & $\pm 0,91$ \\
Orgánica (\%) & 5,12 & $\pm 0,44$ & 5,01 & $\pm 0,41$ & 4,92 & $\pm 0,37$ \\
Acidez (pH) & 0,94 & $\pm 0,04$ & 0,87 & $\pm 0,08$ & 0,86 & $\pm 0,01$ \\
Densidad & & & &
\end{tabular}

SD: desviación estándar

Tabla 2. Caracterización de fungicidas usados en el cultivo de arveja $\mathrm{P}$. sativum (n= 30 fincas) * Fuente: elaboración propia.

\begin{tabular}{|c|c|c|c|c|c|c|}
\hline Ingredienteactivo & CT & Tox. Hombre & $\begin{array}{c}\text { Solubilidad en } \\
\text { Agua (mg L-1) }\end{array}$ & Tox. Abejas & Tox. Peces & Tox. Aves \\
\hline Azoxystrobin & III & NMT & 6 & LT & $\mathrm{ET}$ & NMT \\
\hline Azufre & III & NMT & 0,06 & MT & $\mathrm{ET}$ & LT \\
\hline Benalaxil & III & NMT & 28,6 & $\mathrm{LT}$ & $\mathrm{AT}$ & $\mathrm{LT}$ \\
\hline Benomil & III & $\mathrm{LT}$ & 2,9 & MT & $\mathrm{LT}$ & MT \\
\hline Captan** & II & $\mathrm{AT}$ & 5,1 & $\mathrm{LT}$ & MT & $\mathrm{LT}$ \\
\hline Carbendazim & III & $\mathrm{LT}$ & 8 & $\mathrm{LT}$ & MT & $\mathrm{LT}$ \\
\hline Clorotalonil & II & $\mathrm{AT}$ & 1 & $\mathrm{AT}$ & $\mathrm{AT}$ & $\mathrm{LT}$ \\
\hline Cymoxanil & III & $\mathrm{LT}$ & 780 & MT & MT & $\mathrm{LT}$ \\
\hline Cyprodinil & III & $\mathrm{LT}$ & 16 & NMT & $\mathrm{AT}$ & MT \\
\hline Difeconazol & II & $\mathrm{LT}$ & 15 & $\mathrm{NMT}$ & MT & $\mathrm{LT}$ \\
\hline Dimetomorf & III & $\mathrm{LT}$ & 12 & $\mathrm{LT}$ & MT & $\mathrm{LT}$ \\
\hline Fludioxonil & II & $\mathrm{LT}$ & 2 & $\mathrm{LT}$ & MT & $\mathrm{LT}$ \\
\hline Fluopicolide & III & MT & 3 & $\mathrm{LT}$ & $\mathrm{ET}$ & $\mathrm{LT}$ \\
\hline Flutriafol & II & $\mathrm{LT}$ & - & MT & MT & $\mathrm{LT}$ \\
\hline Folpet** & III & NMT & 0,8 & LT & MT & $\mathrm{LT}$ \\
\hline Iprodione & II & $\mathrm{LT}$ & 12 & $\mathrm{LT}$ & MT & $\mathrm{LT}$ \\
\hline Mancozeb & III & NMT & 6,2 & $\mathrm{LT}$ & $\mathrm{ET}$ & $\mathrm{LT}$ \\
\hline Metalaxil & II & MT & 8,4 & LT & MT & MT \\
\hline Myclobutanil & II & $\mathrm{LT}$ & 132 & $\mathrm{LT}$ & MT & MT \\
\hline Pyrimethanil & II & NMT & 121 & LT & MT & $\mathrm{LT}$ \\
\hline Procloraz & II & $\mathrm{LT}$ & 34,4 & LT & $\mathrm{AT}$ & $\mathrm{LT}$ \\
\hline Procimidona & II & NMT & 2,46 & $\mathrm{LT}$ & $\mathrm{AT}$ & $\mathrm{LT}$ \\
\hline Propamocarb & III & NMT & - & MT & $\mathrm{LT}$ & MT \\
\hline Propiconazol & II & MT & 100 & $\mathrm{LT}$ & MT & $\mathrm{LT}$ \\
\hline Tiram & III & MT & 16,5 & $\mathrm{LT}$ & $\mathrm{ET}$ & $\mathrm{LT}$ \\
\hline
\end{tabular}

*CT: Categoría toxicológica; NMT: No muy tóxico; LT: Ligeramente tóxico; MT: Moderadamente tóxico; AT: Altamente tóxico; ET: Extremadamente tóxico; **: Revaluación o cancelación del registro de venta. 
De estos, el clorotalonil se destaca por su categorización como agente carcinogénico tipo B para el hombre y por presentar toxicidad alta para abejas $\mathrm{y}$ peces de acuerdo a la Red Internacional de Acción en Plaguicidas [16].

De igual forma, los ingredientes activos insecticidas fipronil, cipermetrina, dimetoato, lambda cialotrina y clorpirifos de categoría II de toxicidad y el metomil catalogado dentro del grupo Ib (muy peligroso) presentaron una elevada frecuencia de aplicación en las fincas (Tabla 3). Estas moléculas muestran prominente movilidad en el suelo, potencial contaminante de agua subterránea y todas son altamente tóxicas para las abejas, peces y aves [16].

Los insecticidas anteriores presentan, además, alto riesgo potencial para el hombre al ser carcinogénicos tipo $\mathrm{C}$ y en el caso del dimetoato, clorpirifos y metomil presentan efectos negativos en el sistema nervioso, reproductor y endocrino, que pueden afectar a los campesinos que aplican o están cerca de cultivos de arveja, ya que estas moléculas son inhibidoras de la colinesterasa.

En las jornadas de trabajo con los campesinos, en términos generales, las aplicaciones fueron efectuadas sin el suficiente criterio técnico, con problemas de identificación de la plaga, inadecuada calibración de equipos, condiciones climatológicas variables o desfavorables, afección a organismos benéficos y fueron ausentes las medidas de bioseguridad para el operario.

Siguiendo con la caracterización, el empleo de productos con formulación sólida presentó un uso entre 10,5 a $21,5 \mathrm{~kg} / \mathrm{ha}$, sumado a la aplicación de 20 a 36,4 l/ha de plaguicidas con formulación líquida, tal como se representa en la Fig. 2.

Ante esto, los productores indican un aumento paulatino en las dosis y número de aplicaciones, a partir del momento en el cual el cultivo de arveja se instauró como monocultivo y se intensificó en la región.

Los resultados permiten concluir, a nivel de clúster, que, si bien los tres grupos presentan elevadas cargas en el uso de plaguicidas, son los campesinos con las características del grupo C3, los que emplean un mayor volumen, cuando se extrapola los valores a un área de referencia común (una hectárea) como se observa en la Fig. 2.

$\mathrm{Al}$ respecto se ha indicado que los productores que cultivan en ambientes más desfavorables, suelen extremar las medidas de control de plagas, por lo que utilizan mayores dosis de plaguicidas [17].

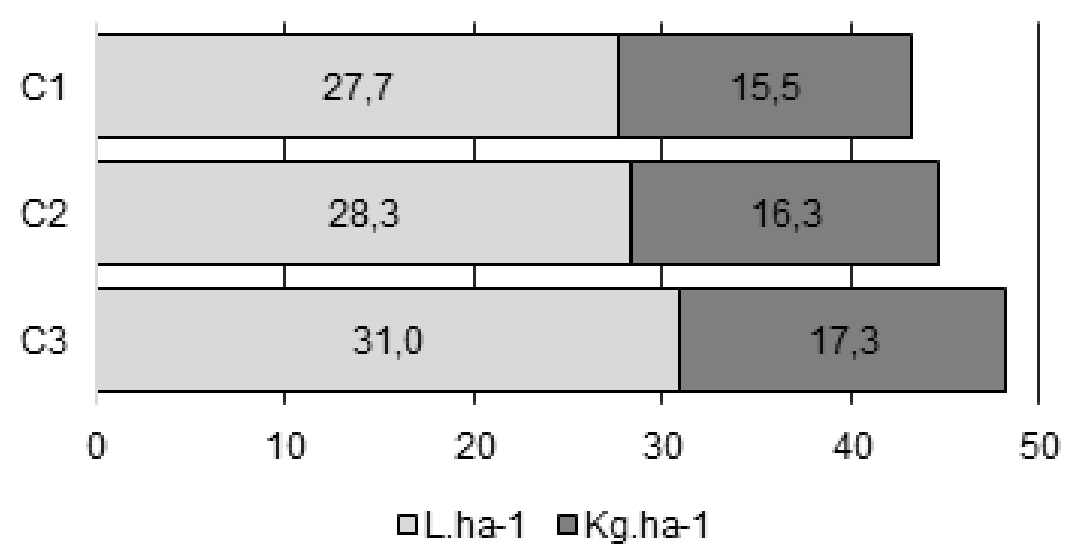

Fig. 2. Estimación del uso ponderado de plaguicidas por hectárea en el cultivo de arveja Fuente: elaboración propia. 
Estimación del riesgo ambiental causado por plaguicidas en cultivos de arveja de Ipiales, Nariño-Colombia

Tabla 3. Caracterización de insecticidas, herbicidas y bactericidas usadas en el cultivo de arveja (P. sativum), $(\mathrm{n}=30$ fincas) *. Fuente: elaboración propia.

\begin{tabular}{|c|c|c|c|c|c|c|}
\hline Ingrediente activo & CT & Tox. Aguda & $\begin{array}{c}\text { Solubilidad en } \\
\text { Agua (mg/L) }\end{array}$ & Tox. Abejas & Tox. Peces & Tox. Aves \\
\hline \multicolumn{7}{|l|}{ Insecticidas } \\
\hline Acefato & III & $\mathrm{LT}$ & 818 & MT & $\mathrm{LT}$ & MT \\
\hline Cipermetrina** & II & $\mathrm{MT}$ & 0,009 & $\mathrm{AT}$ & ET & $\mathrm{LT}$ \\
\hline Ciromazina & III & $\mathrm{LT}$ & 13.600 & $\mathrm{LT}$ & $\mathrm{LT}$ & $\mathrm{LT}$ \\
\hline Clorpirifos $^{* *}$ & II & $\mathrm{MT}$ & 1,18 & $\mathrm{AT}$ & ET & $\mathrm{AT}$ \\
\hline Dimetoato** & II & MT & 39.800 & $\mathrm{AT}$ & MT & $\mathrm{AT}$ \\
\hline Fipronil & II & MT & 22 & $\mathrm{AT}$ & $\mathrm{ET}$ & $\mathrm{AT}$ \\
\hline Indoxcarb & II & $\mathrm{MT}$ & - & $\mathrm{AT}$ & ET & $\mathrm{MT}$ \\
\hline Lamda Cyhalotrin & II & MT & 0,005 & $\mathrm{AT}$ & ET & LT \\
\hline Malation** & III & $\mathrm{MT}$ & 125 & $\mathrm{AT}$ & ET & MT \\
\hline Metamidofos*** & $\mathrm{Ib}$ & $\mathrm{AT}$ & 1.200 .000 & $\mathrm{AT}$ & $\mathrm{MT}$ & $\mathrm{AT}$ \\
\hline Metomyl** & $\mathrm{Ib}$ & $\mathrm{AT}$ & 54.700 & $\mathrm{AT}$ & $\mathrm{ET}$ & $\mathrm{AT}$ \\
\hline Permetrina & II & $\mathrm{MT}$ & 0,006 & $\mathrm{AT}$ & $\mathrm{ET}$ & $\mathrm{LT}$ \\
\hline Profenofos ${ }^{* *}$ & II & $\mathrm{MT}$ & 28 & $\mathrm{AT}$ & ET & MT \\
\hline Tiametoxam & II & & 4.100 & $\mathrm{AT}$ & $\mathrm{LT}$ & MT \\
\hline Triazofos** & $\mathrm{Ib}$ & $\mathrm{AT}$ & 35 & $\mathrm{MT}$ & ET & $\mathrm{ET}$ \\
\hline \multicolumn{7}{|l|}{ Herbicidas } \\
\hline Glifosato & III & $\mathrm{LT}$ & 11.600 & $\mathrm{LT}$ & $\mathrm{AT}$ & $\mathrm{LT}$ \\
\hline Metribuzina & III & MT & 1.030 & $\mathrm{LT}$ & MT & MT \\
\hline Paraquat** & II & MT & 626.000 & MT & MT & MT \\
\hline \multicolumn{7}{|l|}{ Bactericidas } \\
\hline Kasugamicina & III & NMT & - & MT & MT & $\mathrm{LT}$ \\
\hline
\end{tabular}

*CT: categoría toxicológica; NMT: no muy tóxico; LT: ligeramente tóxico; MT: moderadamente tóxico; AT: altamente tóxico; ET: extremadamente tóxico; **: reevaluación o cancelación del registro de venta.

Cabe destacar que el tipo de moléculas usadas por los campesinos de los clústeres C2 y C3 tuvieron en todos los casos una categoría toxicológica mayor a la presentada en $\mathrm{C} 1$, esto incrementa significativamente la posibilidad de producir daño agudo a la salud o al ambiente; se pudo establecer como hipótesis, que los campesinos, con menor capacidad de inversión, seleccionan los plaguicidas a partir del costo en el almacén agrícola, subestimando el efecto nocivo que estos pueden generar.

Respaldando lo anterior, Van der Werf y Zimmer [7] destacan tres factores de selección: el precio del tratamiento, la eficacia esperada y la fitotoxicidad; no es común que se utilice como criterio de compra el nivel de toxicidad por parte del campesino.

Al calcular el uso de insumos en términos de ingrediente activo usando la fórmula de Bravo et al. [11], se demostró que el volumen aplicado en promedio en la muestra evaluada fue de $25,1 \mathrm{~kg}$ ia/ha/año, que podría catalogarse como medio si se compara con otros autores [11], [18], [19], quienes calcularon la cantidad de ingrediente activo en la producción de diferentes cultivos, por ejemplo: forrajes (3,0 kg ia/ha/año), café (3,7 kg ia/ha/año), plátano (5,0 kg ia/ha/año), caña de azúcar $(15,0 \mathrm{~kg}$ ia/ha/año $)$ y arroz $(18,9 \mathrm{~kg}$ ia/ha/año) con una aplicación menor a la calculada para arveja en esta investigación; al mismo tiempo, el uso de insumos fue superado por cultivos más 
intensivos como piña $(25,2 \mathrm{~kg}$ ia/ha/año), papa (42,6 kg ia/ha/año), banano $(49,3 \mathrm{~kg}$ ia/ha/año), tomate $(46,5 \mathrm{~kg}$ ia/ha/año) y melón (60,5 a 74,7 kg ia/ha/año).

Como lo ha demostrado esta caracterización, tanto el ingrediente activo como la dosis aplicada se convierten en elementos de manejo del sistema a tener en cuenta. Sin embargo, ante la complejidad de las situaciones en campo, se justifica el uso de indicadores agregados como lo es I-PHY [6],[20].

\subsection{Cálculo del indicador I-PHY}

Los resultados obtenidos establecieron diferencias en la mayoría de los módulos del indicador a partir de las particularidades de los tres clústeres evaluados, exponiendo las externalidades generadas sobre el ambiente. Ante esto, se advierte que los valores de I-PHY no se pueden tomar como absolutos, pero permiten comparar el riesgo de la transferencia de plaguicidas mediante una escala que facilita la interpretación por parte de los usuarios finales, como campesinos, técnicos e ingenieros agrónomos [12], [20].

En cuanto al módulo de agua subterránea se determinó que en los tres clústeres se presenta una calificación de «riesgo moderado» (Tabla 4), valor obtenido que permite explicar la posibilidad que un plaguicida aplicado al cultivo alcance agua subterránea y afecte su consumo [7]. Las características del entorno, especialmente las relacionadas a la infiltración, incrementaron el riesgo estimado, siendo la granulometría del suelo (textura y estructura), la cubierta vegetal y las prácticas de mecanización inadecuadas, elementos importantes que incrementan la infiltración y con ella el movimiento de plaguicidas [14].

Además, el valor de Iphy-subterránea se incrementa cuando los plaguicidas aplicados tienen ingredientes activos con propiedades fisicoquímicas de alta vida media en el campo (DT50), alta solubilidad en agua, bajo coeficiente de partición orgánico carbono-agua (Koc) y categoría toxicológica alta (CT), elevando el riesgo ambiental percibido en el módulo; este tipo de características estuvieron directamente relacionadas con la selección de los productos por parte del campesino $\mathrm{y}$ permite realizar una evaluación especifica en cada finca para la exclusión de ingredientes activos que generan mayor riesgo ambiental.

Los plaguicidas tienen la capacidad para movilizarse en la matriz del suelo, especialmente cuando son aplicados de forma general en el lote y directamente sobre una matriz disturbada. Si bien la mayor parte de aplicaciones se realiza al follaje del cultivo, se pudo establecer en terreno que las aplicaciones dirigidas al control de arvenses y de insectos barrenadores en las primeras etapas del cultivo para este módulo fueron las más preocupantes.

Tabla 4. Estimación de los módulos del índice I-PHY*. Fuente: elaboración propia.

\begin{tabular}{cccccc}
\hline Clúster & Iphy-Subterr & Iphy-Superf & Iphy-Aire & Iphy-Global & RG \\
\hline C1 & $4,13^{\mathrm{a}}$ & $6,95^{\mathrm{a}}$ & $1,91^{\mathrm{a}}$ & $4,04^{\mathrm{a}}$ & $\mathrm{M}$ \\
$\mathrm{C} 2$ & $4,58^{\mathrm{a}}$ & $9,12^{\mathrm{b}}$ & $1,94^{\mathrm{a}}$ & $5,06^{\mathrm{b}}$ & $\mathrm{A}$ \\
$\mathrm{C} 3$ & $4,80^{\mathrm{a}}$ & $10,0^{\mathrm{b}}$ & $2,52^{\mathrm{a}}$ & $5,28^{\mathrm{b}}$ & $\mathrm{A}$
\end{tabular}

*Medias con una letra diferente en la columna indican diferencias estadísticamente significativas $(\mathrm{P} \leq 0,05)$; RG: riesgo global = M: moderado, A: alto. 
En un estudio similar en la provincia de Santa Fe [17] y en la provincia de Buenos Aires (Argentina) [1], se pudo establecer que los herbicidas son los plaguicidas que mayores problemas ambientales y a la salud humana ocasionan, debido a la combinación de alta toxicidad, alta cantidad aplicada por campaña agrícola y un riesgo de lixiviación mayor que los insecticidas o fungicidas. Coincidiendo con esto, en la caracterización de los ingredientes activos usados en el cultivo de arveja, sobresale por el volumen aplicado el glifosato, paraquat y metribuzina, todos herbicidas; sin embargo, deberá considerarse regular el uso de otras moléculas altamente móviles en el agua como ciromazina, dimetoato, metamidofos, metomil y tiametoxam, los cuales incrementan el peligro latente de contaminación hídrica subterránea (Tabla 3).

Es neurálgico el hecho que un porcentaje de familias campesinas en el municipio de Ipiales usan agua de aljibe para uso doméstico como lo demostró la tipificación previa [8]. Esta agua, de acuerdo a los resultados de este módulo, puede contener trazas de plaguicidas como lo sugiere un estudio realizado en el municipio de Pasto (Nariño), al indicar que estos productos no solo se dispersan en el medio, sino también permanecen en el ambiente por su capacidad de bioacumulación [21], pero además, el uso de acueducto veredal, bajo estas condiciones, no garantiza la calidad del agua, ya que se ha indicado que muchos de los plaguicidas no son fácilmente eliminados por los tratamientos de potabilización, ni siquiera por los más sofisticados.

Por su parte, el módulo Iphy-Superficial mostró un riesgo alto y muy alto de movimiento de los plaguicidas en el campo para alcanzar el agua de estanques, ríos, arroyos y otros, siendo este, el módulo que amerita una intervención prioritaria en la región. El cálculo dependió de cinco variables de entrada, en donde se relacionaron las características fisicoquímicas de los plaguicidas con la inclinación de la pendiente, textura del suelo, cobertura vegetal y distancia a la fuente de agua, en la mayoría de casos con el máximo conflicto bajo las condiciones que se desarrolla el cultivo de arveja.

De acuerdo a la caracterización realizada, los campesinos agrupados en los clústeres $\mathrm{C} 2$ y $\mathrm{C} 3$ aplicaron los plaguicidas más tóxicos, influyendo sobre la calificación de riesgo, que para estos dos casos fue muy alto, de acuerdo a la escala usada (Tabla 4). En estos clústeres, 10 ingredientes activos mostraron toxicidad extrema en peces y 5 tuvieron valores de $\mathrm{DL}_{50}$ oral menores a $50 \mathrm{mg} / \mathrm{kg}$ en ingesta para el hombre, catalogados como altamente y extremadamente tóxicos (Tabla 2 y Tabla 3).

Por otro lado, el módulo Iphy-Aire que evaluó el peligro de contaminación de los plaguicidas después de volatilización, determinó que el riesgo fue bajo (Tabla 4); no obstante, se ha indicado que este es el componente más difícil de estudiar ya que las variables de entrada son relativamente escasas para algunos ingredientes activos, lo cual podría aminorar el riesgo real [7].

A pesar de representar periodos de contacto relativamente cortos, no dejan de ser repetitivos durante la jornada de trabajo, provocando problemas de salud [20], por lo cual, el riesgo de impacto ambiental sobre el aire no puede ser subestimado hasta ser evaluado con métodos específicos.

En cultivos de papa (Solanum tuberosum) y cebolla (Allium cepa), que comparten un manejo similar al identificado para arveja en este estudio, los investigadores pudieron establecer que la tendencia de los campesinos a vivir junto al cultivo, no permite cumplir con el tiempo mínimo de aislamiento post aplicación recomendado y dependiendo de las condiciones climáticas, el viento fue catalogado como un agente que dispersó 
hasta por 500 metros los plaguicidas aplicados en el cultivo; como indican los autores, este tipo de exposición crónica normalmente es ignorada [21].

La comparación y análisis de resultados se centró entonces en el módulo agregado Iphy-Global, el cual compila información de los diferentes módulos con la dosis y frecuencia de aplicación de cada uno de los ingredientes activos, presentando diferencias estadísticas y cualitativas entre $\mathrm{C} 1$ con un riesgo moderado frente a los clústeres $\quad \mathrm{C} 2 \quad \mathrm{y} \quad \mathrm{C} 3, \quad$ los cuales incrementaron en una unidad el valor promedio del indicador, estableciendo un riesgo alto (Tabla 4). Estos resultados concuerdan con el rango presentado por Le Bellec et al. [20], evaluando pequeñas fincas productoras de papa (S. tuberosum) y tomate (Solanum lycopersicum) en la república de Mauricio con un puntaje entre 3,6 y 5,3 de Iphy-global.

La mayoría de investigaciones consultadas, evalúan cultivos más intensivos; por ejemplo, en Argentina, se determinó un rango del módulo agregado en cultivos de maíz a gran escala (Zea mays) con un puntaje entre 4,88 a 5,5 , trigo (Triticum) con 6,42 a 6,82 o soja (Glycine $\max$ ) con 6,39 a 6,82 [22]; igualmente, se han evaluado en Francia y Brasil cultivos comerciales de cebada (Hordeum vulgare) con un puntaje de Iphy-global de 6,07, trigo (Triticum) con 6,47, colza (Brassica napus) con 6,6 , cebolla (Allium) con 6,6 y tabaco (Nicotiana tabacum) con 8,2 [6], superiores al valor promedio determinado en esta investigación para arveja entre 4,04 y 5,28, pero que genera preocupación al ser un cultivo que es catalogado de pequeña escala, con una inversión sumamente menor y con características ambientales que potencializan en todos los casos el riesgo de impacto ambiental (Tabla 4).

Los estudios referenciados sirven para analizar el caso de arveja de Nariño, en donde se puede concluir que el modelo de Revolución verde, en el afán de controlar las plagas y enfermedades, ha llevado en un corto tiempo a incrementar el número de aplicaciones con plaguicidas, sobredosificar y usar productos en periodos y condiciones inadecuadas [17], [20], [22], [23]. Por otro lado, la homogeneidad de los monocultivos en el paisaje agrícola afecta las características de autorregulación inherentes a las comunidades naturales, destruyendo el frágil equilibrio de sus interacciones, al mismo tiempo que favorece una mirada a corto plazo que busca obtener el máximo rendimiento sin considerar la sustentabilidad ambiental, social y económica del sistema.

En vista de ello, se debe cuestionar al paradigma dominante y buscar nuevas vías conceptuales para construir sistemas sustentables, realizando transformaciones progresivas adaptadas al contexto, en donde el enfoque agroecológico ha permitido desarrollar esta conversión en un proceso llamado «transición agroecológica». Para esto, distintos autores han propuesto como primera medida incrementar la eficiencia del uso de insumos y paralelamente realizar una sustitución de plaguicidas altamente tóxicos con alternativas de manejo de menor riesgo para el ambiente [20], [24].

Paulatinamente, se le debe integrar estrategias de manejo de problemas fitosanitarios, por ejemplo, el diseño y uso de umbrales, la sustitución de herbicidas por laboreo manual o uso de coberturas vegetales, protección del suelo, uso de cultivos trampa o barrera, aplicación de productos biológicos, entre otros.

En este estudio se aporta a esta fase de transición, al analizar el sistema de arveja a través de una herramienta de monitoreo basada en el indicador I-PHY, que permite desde la investigación participativa calificar el riesgo de impacto ambiental a nivel de finca y clúster, para generar a futuro estrategias de manejo, como se ha demostrado en otras investigaciones [20], [23], al establecer que este indicador permite desarrollar un método eficiente de 
aplicación de productos y tomar decisiones en la selección o sustracción de ingredientes activos de acuerdo al contexto de producción.

Otro resultado importante encontrado al analizar el indicador I-PHY fue la relación que se establece con el contenido de materia orgánica determinado en cada finca; niveles adecuados contribuyeron a la reducción de la probabilidad de transferencia de los ingredientes activos hacia los cursos de agua disminuyendo el valor calculado. $\mathrm{Al}$ respecto, se ha indicado en otros estudios que el incremento de la materia orgánica influye directa e indirectamente sobre los atributos físicos, químicos y biológicos del suelo, relacionados con la adsorción-desorción de plaguicidas y con la degradación atribuida a la actividad microbiana, catalogado como el mecanismo de descomposición más importante en el ambiente [1]. Por tanto, la formulación de estrategias integradas que permitan la recuperación, conservación y manejo del suelo en la finca, aumentando paulatinamente el reciclado de biomasa y la actividad biótica, junto a la gestión de insumos podrían ser las primeras estrategias para aminorar los valores de riesgo obtenidos.

Sin embargo, no se puede olvidar que el rediseño del sistema es la última parte del proceso que induce necesariamente a un cambio de paradigma y giro hacia el desarrollo de sistemas de cultivo agroecológicos, en los cuales los servicios ecosistémicos remplazan las entradas sintéticas, preponderando la restauración de la fertilidad del suelo, la lucha biológica por la conservación de hábitat y el incremento de interacciones benéficas.

Esta es la forma profunda de una reconversión agroecológica, en donde el campesino y su familia son el centro del proceso, creando una construcción mancomunada que permite diseñar sistemas resilientes que tengan un bajo impacto [20], incidiendo sobre la responsabilidad intergeneracional de preservación del ambiente y del potencial productivo del sistema.

\section{CONCLUSIONES}

En la muestra evaluada se identificaron 44 ingredientes activos con un promedio de uso de $25,1 \mathrm{~kg}$ ia/ha/año, la mayor proporción de productos fueron fungicidas con el $59 \%$, insecticidas $29 \%$, herbicidas $8 \%$ y bactericidas $5 \%$, mostrando una alta dependencia de plaguicidas en el sistema productivo de arveja.

A partir del módulo I-phy-Global se pudo determinar que en la región existe un riesgo de contaminación ambiental variable, dependiendo del tipo de productor, presentando un riesgo moderado para los campesinos agrupados en $\mathrm{C} 1(4,04)$ y un riesgo alto para los campesinos agrupados en C2 $(5,06)$ y C3 $(5,28)$. La contaminación de fuentes hídricas superficiales se convierte en el mayor conflicto por uso de plaguicidas en el cultivo de arveja.

\section{CONFLICTO DE INTERESES}

Los autores declaran que no presentan conflicto de intereses.

\section{AGRADECIMIENTOS}

Los autores agradecen a la Gobernación de Nariño y al Centro de Excelencia CeiBA, al permitir el desarrollo de esta investigación.

\section{REFERENCIAS}

[1] F. Bedmar, V. Gianelli, H. Angelini, y L. Viglianchino, "Riesgo de contaminación del agua subterránea con plaguicidas en la cuenca del arroyo El Cardalito, Argentina," Rev. Investig. Agropecu., vol. 41, no. 1, pp. 70-82, Apr. 2015. Disponibel en: URL 
Estimación del riesgo ambiental causado por plaguicidas en cultivos de arveja de Ipiales, Nariño-Colombia

[2] F. Le Bellec, A. Vélu, L. Squin, and T. Michels, "Utilisation de l'indicateur I-PHY comme outil d'aide à la décision en verger d'agrumes à la Réunion -Le cas de la lambdacyhalothrine," Innov. Agron., vol. 31, pp. 6173, Oct. 2013. Disponible en: URL

[3] C. A. Vela-Monroy, M. J. Saavedra-Alemán, and J. G. Carriazo-Baños, "Catalizadores homogéneos y heterogéneos de $\mathrm{Fe} 3+, \mathrm{Co} 2+\mathrm{y}$ $\mathrm{Cu} 2+$ para la degradación de metilparatión en medio acuoso diluido," TecnoLógicas, vol. 19 , no. 37 , pp. 13-28, Jul. 2016. https://doi.org/10.22430/22565337.43

[4] M. A. Altieri y C. I. Nicholls, "Agroecología: única esperanza para la soberanía alimentaria y la resiliencia socioecológica," Agroecología, vol. 7, no. 2, pp. 65-83, Dec. 2012. Disponible en: URL

[5] D. Grenón, E. Galán, F. Pernuzzi, and S. Guzman, "IIRAmb: avances en el Índice Integrado de Riesgo Ambiental por el uso de plaguicidas en cultivos," en 47 JAIIO CAI, Congreso Argentino de AgroInformática, Palermo, 2015, pp. 127-139.

Disponible en: URL

[6] C. L. Beber et al., "Comparative agroenvironmental risks of pesticides in different cropping systems: application of the I-Phy indicator," Environ. Earth Sci., vol. 77, no. 532, pp. 1-10, Jul. 2018. https://doi.org/10.1007/s12665-018-7698-6

[7] H. M. G. van der Werf y C. Zimmer, "An indicator of pesticide environmental impact based on a fuzzy expert system," Chemosphere, vol. 36, no. 10, pp. 2225 - 2249, Apr. 1998.

https://doi.org/10.1016/S0045-6535(97)101941

[8] D. E. Alvarez-Sánchez, E. D. Gómez-López, y H. R. Ordóñez-Hurtado, "Tipología de fincas productoras de arveja (Pisum sativum L.) en la subregión Sur de Nariño, Colombia," Cienc. y Tecnol. Agropecu., vol. 20, no. 3, pp. 659-677, Sep. 2019.

https://doi.org/10.21930/rcta.vol20_num3_art $\underline{: 1593}$

[9] Agronet Ministerio de Agricultura, "Área cosechada, producción y rendimiento de arveja, 1986-2017," Unión Temporal Crece Federación, Colombia, reporte, 2018. Disponible en: URL

[10] Instituto Geográfico Agustin Codazzi (IGAC), "Diccionario Geográfico de Colombia," 2015. Disponible en: URL

[11] V. Bravo-Durán, E. De la Cruz-Malavasii, G. Herrera-Ledezma, y F. Ramirez-Muñoz, "Uso de plaguicidas en cultivos agricolas como herramienta para el monitoreo de peligros en salud," Uniciencia, vol. 27, no. 1, pp. 351-376, Jan. 2013. Disponible en: URL
[12] M. C. Arregui, D. Grenón, D. Sánchez y J. Ghione, "Evaluación del riesgo de impacto ambiental de plaguicidas en cultivos anuales del centro de Santa Fe," Fave. Sección ciencias Agrar., vol. 12, no. 1/2, pp. 13-21, Dec. 2013.

https://doi.org/10.14409/fa.v12i1/2.5114

[13] H. D. Mena, J. A. Castillo, y J. A. Marín, "Resultados preliminares sobre la calibracion de un kit portatil para análisis químico de suelos," Rev. Ciencias Agrícolas, vol. 30, no. 1, pp. 79-86, 2013. Disponible en: URL

[14] D. Jaramillo, "Introducción a la ciencia del suelo," (Documento de trabajo), Escuela de Geociencias y Medio Ambiente, Universidad Nacional de Colombia, 2002. Disponible en: URL

[15] Instituto Colombiano Agropecuario. ICA, "Registros nacionales de plaguicidas de uso agrícola registrados ante el ICA, noviembre de 2019," .Disponible en: URL

[16] Pesticide Action Network, "PAN Pesticides Database - Pesticide Products," Pesticide Action Network, 2017. Disponible en: URL

[17] S. Montico y N. Di Leo, "Riesgo ambiental por pesticidas en una cuenca del sur de la provincia de Santa Fe, Argentina," Rev. Int. Contam. Ambient., vol. 31, no. 2, pp. 165-172, Aug. 2015. Disponible en: URL

[18] L. Abraham, L. Alturria, A. Fonzar, A. Ceresa y E. Arnes, "Propuesta de indicadores de sustentabilidad para la producción de vid en Mendoza, Argentina," Rev. la Fac. Ciencias Agrar., vol. 46, no. 1, pp. 161-180, Jun. 2014. Disponible en: URL

[19] F. Ramírez-Muñoz, M. L. Fournier-Leiva, C. Ruepert, y C. Hidalgo-Ardón, "Uso de agroquímicos en el cultivo de papa en Pacayas, Cartago, Costa Rica," Agron. Mesoam., vol. 25, no. 2, pp. 337-345, Jul. 2014.

https://doi.org/10.15517/am.v25i2.15441

[20] F. Le Bellec, M. Scorbiac y J. Sauzier, "Les pratiques phytosanitaires des producteurs de légumes de l'île Maurice: Impacts et perspectives de changement," Cah. Agric., vol. 26 , no. 5, pp. 1-9, Sep. 2017.

https://doi.org/10.1051/cagri/2017038

[21] C. Jiménez-Quintero, A. Pantoja-Estrada, y H. Leonel, "Riesgos en la salud de agricultores por uso y manejo de plaguicidas, microcuenca 'La Pila," Univ. y Salud, vol. 18, no. 3, pp. 417-431, Dec. 2016. https://doi.org/10.22267/rus.161803.48

[22] S. Montico, J. Denoia, y J. Berardi, "Impacto y riesgo ambiental del uso de pesticidas en cultivos de la cuenca del Arroyo Ludueña, Santa Fe," Cuad. del Curiham, vol. 20, pp. 73-79, 2014. Disponible en: URL 
Estimación del riesgo ambiental causado por plaguicidas en cultivos de arveja de Ipiales, Nariño-Colombia

[23] F. Le Bellec et al., "Helping farmers to reduce herbicide environmental impacts," Ecol. Indic., vol. 54, pp. 207-216, Jul. 2015. https://doi.org/10.1016/j.ecolind.2015.02.020
[24] C. I. Nicholls, M. A. Altieri, y L. L. Vázquez, "Agroecología: Principios para la conversión y el rediseño de sistemas agrícolas," Agroecologia, vol. 10, no. 1, pp. 61-72, Jun. 2015. Disponible en: URL 